DOI: 10.12957/demetra.2016.22510

\title{
Idealização, marcos e trajetórias de peso em mulheres com excesso de peso: reflexões para o tratamento nutricional
}

\author{
Idealizations and weight trajectories in overweight women: reflections for nutritional \\ treatment
}

Camila de Souza Meirelles'

Lucas Vieira Francisco ${ }^{\top}$

Ana Carolina de Aguiar Moreira'

Rosa Wanda Diez-Garcial

1 Universidade de São Paulo, Faculdade de Medicina de Ribeirão Preto, Departamento de Clínica Médica, Laboratório de Práticas e Comportamento Alimentares - PráticA. Ribeirão Preto-SP, Brasil.

Correspondência / Correspondence

Rosa Wanda Diez-Garcia

E-mail: wanda@fmrp.usp.br

\section{Resumo}

Este estudo exploratório teve o objetivo de compreender a idealização, os marcos e as trajetórias de peso de mulheres com excesso de peso que buscam o tratamento para emagrecer em ambulatório de nutrição. Para isso, comparou-se o peso aferido, o habitual e o desejado de 167 mulheres com excesso de peso atendidas ambulatoriamente em hospital terciário e também analisou-se as informações sobre as causas e a trajetória de alterações de peso registradas com detalhes em 31 prontuários. Os resultados mostraram que as pacientes chegaram ao serviço com obesidade grau II, que é superior ao referido como IMC habitual (obesidade grau I), e desejavam atingir o IMC classificado como sobrepeso. Ao analisar os marcos de ganho de peso foi visto que a maioria deles são eventos que promovem mudanças no contexto social e alimentar, como casamento, alteração na rotina de trabalho, gravidez, doença e problemas familiares. A flutuação de peso (efeito ioiô) em diferentes fases da vida foi recorrente nas descrições do histórico de peso. Majoritariamente, as pacientes $(65,4 \%)$ relataram que anteriormente ao início do tratamento nutricional já haviam realizado dietas para perda de peso sem orientação profissional. Através das análises dos marcos e trajetórias de peso das pacientes é possível verificar uma grande diversidade de fatores que influenciam cada paciente em sua construção social do corpo. Desse modo, acredita-se que a investigação e o entendimento dessas informações podem favorecer a adesão ao tratamento nutricional no contexto clínico de estabelecimento de metas para perda de peso.

Palavras-chave: Obesidade. Mulheres. Alterações do Peso Corporal. Perda de peso. Ganho de peso. 


\section{Abstract}

This exploratory study aimed to understand weight idealization, landmarks and trajectories of overweight women who seek treatment for weight loss in outpatient nutrition clinics. For this purpose, we compared measured weight, usual weight and desired weight of 167 overweight women treated in an outpatient clinic located at a tertiary hospital. We also analyzed the information about the causes and the trajectory of weight changes recorded in 31 detailed records. The results showed that when the patients arrived at the service, they had class II obesity, which is higher than the one usually referred as BMI (class I obesity); also, they desired to reach a BMI classified as overweight. Most of the weight gain landmarks analyzed are events that promote changes in the social and eating context such as marriage, change in work routine, pregnancy, illness and family problems. Weight fluctuation (yo-yo effect) in different stages of life was recurrent in their weight history. Most patients (65.4\%) reported that before starting the nutritional treatment, they held diets to weight loss without professional guidance. Through the analysis of patients' landmarks and weight trajectories, we could verify the wide range of factors that influence the social construction of the body of each patient. Thereby, it is believed that research and understanding of this information can promote better adherence to nutritional treatment in the clinical context of establishing goals for weight loss.

Key words: Obesity. Women. Body weight changes. Weight loss. Weight gain. Diet.

\section{Introdução}

O excesso de peso na população é resultante de um dinâmico e complexo processo que envolve mudanças alimentares que ocorrem ao longo da vida, as quais refletem a história pessoal e os papéis sociais, convivendo com os padrões estéticos idealizados e os socialmente aceitos. ${ }^{1-3}$

Tratamentos nutricionais são frequentemente voltados para o peso ideal, embora muitos estudos reconheçam a importância da satisfação do paciente em relação à meta de tratamento. ${ }^{2,4}$ e que a perda de $5 \%$ a $10 \%$ do peso já apresenta benefícios metabólicos, como o controle glicêmico, redução da pressão arterial e níveis de colesterol e melhora da qualidade de vida..$^{5-8}$ 
Percebemos o mundo não apenas através dos sentidos, mas também pelos significados sociais e culturais que atribuímos a ele. $\mathrm{O}$ modo como percebemos nosso próprio corpo é influenciado por fatores sociais e culturais em um determinado contexto histórico. ${ }^{9,10}$ A convivência dentro de um grupo molda como idealizamos o corpo e, consequentemente, como o desejamos, visto que normas e padrões de referência são modificados através das relações sociais. ${ }^{11,12}$

Essas percepções e idealizações aplicam-se também às construções do conceito de peso e corpo. ${ }^{9}$ Muitas vezes pacientes relatam concepções de peso que não condizem com a realidade. ${ }^{12-14}$ Esta distorção na percepção e idealização de peso pode dificultar a adoção de atitudes relacionadas à perda de peso e afetar a motivação para o tratamento., ${ }^{45}$ A frustração em não alcançar o peso idealizado pelos próprios pacientes pode também ser um fator desmotivador. ${ }^{16,17}$

Desse modo, a idealização do peso e as metas de perda de peso desejadas pelos pacientes são aspectos que ganham importância no tratamento nutricional. Maynard et al. analisaram tendências de peso desejado nos EUA entre 1994 e 2003 e concluíram que mulheres com obesidade desejam um peso entre $15 \%$ e $25 \%$ menor que o atual. ${ }^{12} \mathrm{O}$ estudo de Foster et al. concluiu que mulheres que procuram tratamento desejam uma perda de peso de $32 \%$ do atual e uma perda em torno de $17 \%$ foi considerada insatisfatória. ${ }^{13}$

A história de peso é outro componente importante para o tratamento, visto que este relato está ligado ao modo pelo qual o paciente percebe e registra o ganho de peso..$^{18,19}$

O movimento cíclico de peso, também conhecido na literatura como peso flutuante, efeito "ioiô" ou "sanfona", é um elemento presente na história de peso, sendo constituído por perda de peso intencional e posterior ganho de peso ou vice e versa. Andrade e colaboradores encontraram 28,4\% de peso flutuante em pacientes assistidos em ambulatório de obesidade, variando o percentual de peso entre $10 \%$ e $35 \%$ do peso inicial, e a taxa de recuperação de peso é de aproximadamente $28,6 \% .^{20}$

Os parâmetros para caracterizar esta condição cíclica em termos de magnitudes de mudança de pesos não são consensuais na literatura. ${ }^{20-22} \mathrm{O}$ estudo de Schulz et al. ${ }^{23}$ concluiu que mudanças de peso em curtos períodos de tempo têm impacto substancial no desenvolvimento da hipertensão arterial sistêmica.

As mudanças de peso podem ser compreendidas em diferentes contextos que impactam na construção social do corpo para paciente. Nesse sentido, faz-se necessário compreender como pacientes com excesso de peso percebem a trajetória de ganho de peso e como idealizam o peso desejado. Assim sendo, o objetivo deste estudo é descrever as trajetórias e as idealizações de peso em mulheres com excesso de peso que buscam tratamento nutricional. 


\section{Metodologia}

Trata-se de um estudo exploratório com metodologia mista, no qual os dados quantitativos estão relacionados aos dados socioeconômicos, o peso aferido, o desejado e o habitual, e a abordagem qualitativa se refere às trajetórias e aos marcos de ganho de peso.

Foram estudadas todas as mulheres com desordens endócrino-ginecológicas associadas ao sobrepeso e obesidade atendidas no Ambulatório de Nutrição da Endocrinologia Ginecológica do Hospital das Clínicas da Faculdade de Medicina de Ribeirão Preto, no período entre 2007 e 2015.

O projeto foi aprovado pelo Comitê de Ética e Pesquisa da Faculdade de Medicina de Ribeirão Preto sob o protocolo 10137/2010.

\section{Dados quantitativos: Caracterização da amostra, Padrão corporal e Idealização}

A caracterização da amostra foi realizada através de um questionário sociodemográfico que continha informações sobre a idade, estado civil, escolaridade, renda per capita, número de filhos e desejo de engravidar neste momento.

O peso aferido (PA) foi obtido por uma balança digital (marca Filizola ${ }^{\circledR}$ ) com capacidade de 150 quilos (kg) e precisão de 100 gramas (g), e a estatura foi medida em metros através do estadiômetro da própria balança. O Índice de Massa Corporal (IMC) foi calculado pela razão do peso $(\mathrm{kg})$ e a estatura $(\mathrm{m})$ ao quadrado e classificado segundo a Organização Mundial de Saúde. ${ }^{16}$

O peso habitual (PH) e peso desejado (PD) foram coletados através de perguntas abertas feitas no início do atendimento.

As análises estatísticas foram realizadas através do software SPSS versão 17.0. Para caracterização da amostra, descrição da flutuação de peso prévio ao tratamento e o peso aferido (PA), peso habitual (PH) e peso desejado (PD) foram utilizados média, desvio padrão e frequência percentual. Para comparar os pesos aferido, habitual e desejado foram aplicados o teste de análise variância (ANOVA), seguido pelo Test T de Student, para amostras pareadas, considerando o efeito de significância estatística de $\mathrm{p}<0,05$.

\section{Dados qualitativos: Trajetória e Marcos do ganho de peso}

Os dados qualitativos tiveram como objetivo caracterizar a percepção das pacientes sobre a evolução de peso no decorrer da vida, identificando os principais marcos do ganho de peso e as tentativas de perda de peso prévias ao tratamento nutricional. 
Para isso, avaliou-se os seguintes tópicos no prontuário de atendimento: i) "evolução do peso corporal", que investiga a história das mudanças do peso durante toda a vida e as possíveis causas da sua variação (peso na infância, adolescência, gestação, entre outras fases da vida); ii) "tratamentos anteriores", que investiga as possíveis tentativas de perda de peso prévias ao tratamento atual (dietas, ajuda profissional, atividade física, medicamentos, entre outros métodos).

As anotações sobre essas respostas foram analisadas recorrendo à proposta de método de Braun e Clark. ${ }^{24}$ As palavras ou trechos, quando relatados exatamente como a paciente referiu durante a consulta, foram colocados entre aspas e cada citação será seguida pela letra $\mathrm{P}$ (paciente).

\section{Resultados}

\section{Caracterização da amostra}

A amostra foi composta por 167 mulheres, com idade entre 18 e 42 anos (média de 29,7 $\pm 6,4$ anos). Entre essas mulheres, quase $70 \%$ não tinham filhos e $53 \%$ mostraram desejo de engravidar. Aproximadamente $75 \%$ das pacientes apresentavam a síndrome do ovário policístico como desordem endócrino-ginecológica, associada principalmente às seguintes comorbidades: síndrome metabólica, diabetes mellitus, hipertensão arterial, hipotireoidismo e dislipidemia. Noventa por cento das pacientes apresentam obesidade $(n=152)$, das quais $61 \%$, classificação de IMC superior a $34,9 \mathrm{~kg} / \mathrm{m}^{2}(\mathrm{n}=102)$. Na tabela 1 podemos observar as características sociodemográficas das pacientes. 
Tabela 1. Características sociodemográfica dos pacientes. Ribeirão Preto, SP - 2016.

\begin{tabular}{lc}
\hline \multicolumn{1}{c}{ Variáveis } & $\%$ \\
\hline Idade (anos) & 29.7 \\
Média & $18-42$ \\
Min - Máx & \\
Estado Civil & 106 \\
Casada & 28 \\
Solteira & 13 \\
Outros & \\
Escolaridade & 33,6 \\
Até 8 anos de estudo & 58,0 \\
De 8 a 11 anos de estudo & 8,3 \\
Superior a 8 anos de estudo & \\
Renda per capita (reais) & \\
Mediana & 5,9 \\
Min - Máx & 29,9 \\
IMC (kg/m ${ }^{2}$ ) & 36,5 \\
25-29,9 & $100-2000$ \\
30-34,9 & \\
35-39,9 & \\
\hline 40 & \\
\hline
\end{tabular}

Padrão corporal, peso atual e a idealização de corpo

Baseado nos pesos e altura avaliados, foram calculados os IMC referentes a cada variável. As médias e os erros padrões estão apresentados na figura 1. Nota-se que a média do IMC do peso aferido $\left(37,1 \pm 6,1 \mathrm{~kg} / \mathrm{m}^{2}\right)$ é classificada como obesidade grau II, a média de IMC do peso habitual, como grau I $\left(32,3 \pm 7,4 \mathrm{~kg} / \mathrm{m}^{2}\right)$, enquanto a média de IMC do peso desejado, como sobrepeso $\left(26,7 \pm 3,4 \mathrm{~kg} / \mathrm{m}^{2}\right)$. Observa-se que todas as médias dos valores de IMC são diferentes entre si $\left(\mathrm{M}_{\mathrm{PA}}: 37,1 \pm 6,1 ; \mathrm{M}_{\mathrm{PH}}: 32,3 \pm 7,4 \mathrm{~kg} ; \mathrm{M}_{\mathrm{D}}: 26,7 \pm 3,4 \mathrm{~kg} / \mathrm{m}^{2} \mid \mathrm{PA} \times \mathrm{PH} \mathrm{F}(1,323)=38,79 ; \mathrm{p} \leq 0,000 \mathrm{~T}\right.$ $(159)=9,73 ; \mathrm{p} \leq 0,000|\mathrm{PA} \times \mathrm{PD} F(1,323)=73,44 ; \mathrm{p} \leq 0,000 \mathrm{~T}(159)=10,23 ; \mathrm{p} \leq 0,000| \mathrm{P} \times$ PD F $(1,323)=344,8 ; \mathrm{p} \leq 0,000 \mathrm{~T}(159)=24,72 ; \mathrm{p} \leq 0,000)$. 


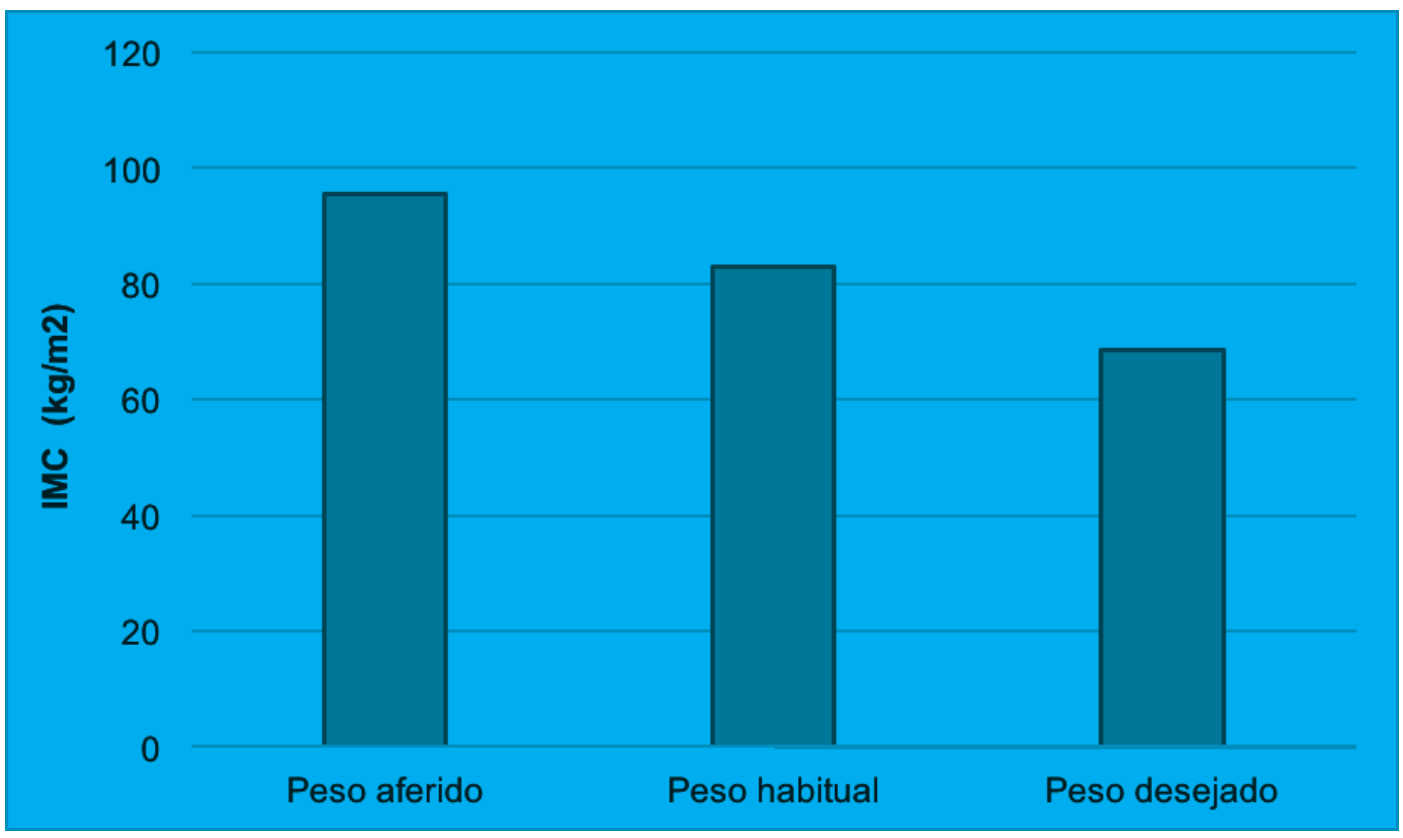

Figura 1. Comparação entre as médias e erros padrões do Índice de Massa Corporal (IMC) calculados com pesos aferido, peso habitual e peso desejado. Ribeirão Preto - SP, 2016.

Ao analisar o peso aferido com o peso habitual relatado pelas pacientes (PA x PH), foi visto que $54,8 \%(\mathrm{n}=90)$ das mulheres relataram peso habitual superior ao peso aferido $(\geq 105 \%$ do peso aferido), $34,1 \%$ ( $\mathrm{n}=56$ ), peso habitual semelhante ao peso aferido (94,9\% a 104,9\% do peso aferido) e 7,9\% ( $n=13)$, peso habitual inferior ao peso aferido ( $\leq 95 \%$ do peso aferido). As análises estáticas mostraram diferença significativa entre esses dois pesos (aferido e habitual) na amostra estudada $\left(\mathrm{M}_{\mathrm{PA}}: 95,6 \pm 18,0 \mathrm{~kg} ; \mathrm{M}_{\mathrm{PH}}: 82,9 \pm 11,1 \mathrm{~kg} ; \mathrm{F}(1,323)=31,3 ; \mathrm{p} \leq 0,000 \mathrm{~T}(166)=8,24 ; \mathrm{p} \leq 0,000\right)$.

Ao analisar o peso habitual com o peso desejado (PH x PD), a maioria das pacientes (64,6\%) desejavam ter o peso inferior ao peso habitual ( $\leq 95 \%$ do peso habitual), 21,9\%, apresentar peso semelhante ao peso habitual (94,9\% a 104,9\% do peso habitual) e 11,5\% relataram peso desejado superior ao peso habitual (105-140\% do peso habitual). As análises estáticas mostraram diferença significativa entre esses dois pesos (habitual e desejado) na amostra estudada $\left(\mathrm{M}_{\mathrm{PH}}: 82,9 \pm 11,1 \mathrm{~kg}\right.$; $\left.\mathrm{M}_{\mathrm{PD}}: 68,8 \pm 10,1 \mathrm{~kg} ; \mathrm{F}(1,323)=64,9 ; \mathrm{p} \leq 0,000 \mathrm{~T}(166)=10,1 ; \mathrm{p} \leq 0,000\right)$.

E por fim, ao comparar o peso aferido com o peso desejado (PA x PD), observou-se que 166 pacientes $(99,3 \%)$ relataram vontade de perda de peso. Dessas, 65,8\% $(\mathrm{n}=109)$ desejavam perder entre $25 \%$ e $50 \%$ do peso aferido e as demais, entre $6 \%$ e $24,9 \%$ do peso aferido. As análises 
estáticas mostraram diferença significativa entre esses dois pesos (aferido e desejado) na amostra estudada $\left(\mathrm{M}_{\mathrm{PA}}: 95,6 \pm 18,0 \mathrm{~kg} ; \mathrm{M}_{\mathrm{PD}}: 68,8 \pm 12,8 \mathrm{~kg} ; \mathrm{F}(1,323)=280,3 ; \mathrm{p} \leq 0,000 \mathrm{~T}(166)=24,3\right.$; $\mathrm{p} \leq 0,000)$. Na figura 2 estão expostos as frequências dos IMC referentes a cada peso avaliado.

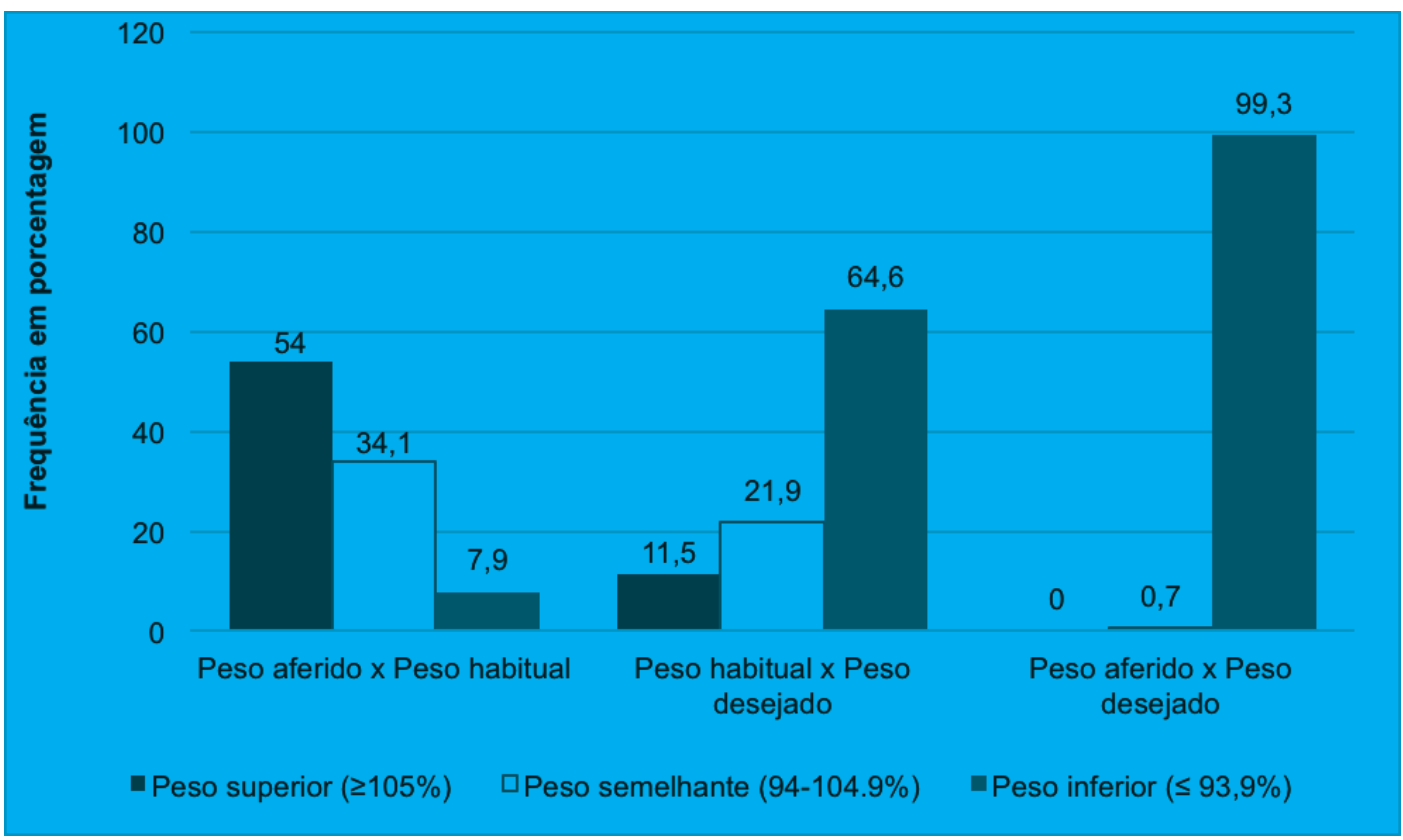

Figura 2. Frequência das pacientes nas comparações entre o peso aferido, peso habitual e peso desejado, considerando as categorias: peso maior ou igual a 105\%, peso semelhante (94-104.5\%) e peso inferior ou igual a 93,9\%. Ribeirão Preto - SP, 2016.

\section{Trajetória e marcos do ganho de peso}

Para análise qualitativa, foram selecionados os prontuários que continham o detalhamento das informações necessárias para realização da investigação proposta (marcos de ganho de peso e flutuação de peso corporal prévio), totalizando 31 prontuários.

Na história de peso, destaque-se a magnitude dos valores de peso associados a um marco, como nos seguintes trechos: "na última gravidez engordou 20 kg", "10 kg em 3 anos" e "15 kg em 8 meses". A variação do ganho de peso relatada foi entre 10 e $40 \mathrm{~kg}$.

A infância, a gravidez e o casamento são os principais marcos temporais referidos para o início do ganho de peso, os quais estão atrelados às mudanças na vida pessoal. Na tabela 2 estão apresentados os motivos e exemplos relatados. 
Tabela 2. Motivos de ganho de peso descritos pelos pacientes e registrados em prontuários por alunos e nutricionistas do HC-FMRP (n=31). Ribeirão Preto - SP, 2016.

\begin{tabular}{|c|c|}
\hline Motivos & Exemplos de trechos relatados \\
\hline \multirow{3}{*}{$\begin{array}{l}\text { Excesso de peso } \\
\text { desde a infância }\end{array}$} & Na infância paciente relata já ser obesa. (P8) \\
\hline & $\begin{array}{l}\text { Refere ter nascido acima do peso }(5 \mathrm{~kg}) \text { e ter sido uma criança } \\
\text { obesa. (P25) }\end{array}$ \\
\hline & Desde criança era além do peso. (P21) \\
\hline
\end{tabular}

Gravidez

"Magra" até 18 anos e após o primeiro filho, 22 anos, engordou $20 \mathrm{~kg} \cdot(\mathrm{Pl})$

Na última gravidez engordou 20 kg e não conseguiu perder. (P16)

Casamento

Após o casamento (aos 18 anos) veio ganhando peso aos poucos, depois começou a comer mais vezes. (P7)

Paciente casou-se aos 16 anos (75 kg) e nos 15 anos posteriores obteve um ganho ponderal de $45 \mathrm{~kg}$. (P2)

Refere peso de $96 \mathrm{~kg}$ em 2009 atingido após quatro anos do casamento, período no qual não realizava atividade física e aumentou ingestão calórica. (P19)

Uso de

medicamentos

Acometimento por uma doença
Relata sempre ter sido magra; em 2000, começou a tomar "hormônio" e engordou 25 kg em 3 meses. (P18)

Pesava 58/59 kg quando tinha 29 anos e passou a ganhar peso quando passou a usar o "remédio". (P9)

Começou o ganho de peso há 6 anos, quando parou o uso de anticoncepcional. (P10)

Há dois anos teve "problema de hérnia de disco", parou atividade física e desde então vem ganhando peso. (P20)

Relatou ter ganho de peso após o diagnóstico. (P9) 


\begin{tabular}{|c|c|}
\hline Motivos & Exemplos de trechos relatados \\
\hline \multirow[t]{3}{*}{ Mudança de vida } & $\begin{array}{l}\text { Quando tinha } 7 \text { anos, após a menstruação, começaram a aparecer } \\
\text { os sintomas (pelos) que fizeram com que as pessoas se afastassem } \\
\text { dela, por isso começou a comer mais e ganhou mais peso. (P17) }\end{array}$ \\
\hline & $\begin{array}{l}\text { Relata que houve mudanças na sua vida (mudou de casa, emprego } \\
\text { e obteve habilitação, tornando-se mais sedentária). (P3) }\end{array}$ \\
\hline & $\begin{array}{l}\text { Ganhou } 20 \mathrm{~kg} \text { até os } 19 \text { anos porque parou de fazer atividade } \\
\text { física. (P23) }\end{array}$ \\
\hline \multirow[t]{2}{*}{ Rotina de trabalho } & $\begin{array}{l}\text { Associa o ganho de peso à ansiedade e ao nervosismo relacionados } \\
\text { ao trabalho. (P30) }\end{array}$ \\
\hline & $\begin{array}{l}\text { Há } 8 \text { anos o ganho de peso se acentuou por mudança da rotina, } \\
\text { começou a trabalhar à noite. (P21) }\end{array}$ \\
\hline \multirow[t]{2}{*}{ Problemas familiares } & Voltou aos 120 kg após a morte de sua mãe. (P15) \\
\hline & $\begin{array}{l}\text { Relata que começou a ganhar peso aos } 9 \text { anos após a separação } \\
\text { de seus pais. (P29) }\end{array}$ \\
\hline
\end{tabular}

P: participante.

O efeito ioiô foi recorrente nas descrições do histórico de peso. Há histórias de ganhos de peso que somam valores consideráveis, seguidos de perda de peso ínfima. Por exemplo, uma paciente refere perda de peso de $15 \mathrm{~kg}$ e posterior ganho de $37 \mathrm{~kg}$ em um ano. Outra paciente refere ter sido "magra" até os 18 anos e com a gravidez do primeiro filho apresentou ganho de $20 \mathrm{~kg}$, seguido de perda de peso, porém, após o falecimento do pai, ganhou $27 \mathrm{~kg}$ e perdeu $4 \mathrm{~kg}$ com uso de medicação.

Por meio dos relatos descritos, é possível observar que mais da metade das pacientes $(\mathrm{n}=18)$ apresentam perda de peso prévia ao tratamento atual. Dessas, $77 \%(\mathrm{n}=14)$ referem perda e ganho de peso (efeito ioiô) (Tabela 3). 
Tabela 3. Registros de relatos de mudança de peso observada entre os pacientes $(n=31)$. Ribeirão Preto - SP, 2016.

\begin{tabular}{ccc}
\hline & $\mathrm{N}$ & $\%$ \\
\hline Relato de perda de peso anterior & 18 & 58,0 \\
Com ganho de peso & 14 & 77,0 \\
Sem descrição de ganho posterior & 4 & 23,0 \\
Não relatam perda de peso & 13 & 42,0 \\
\hline
\end{tabular}

Os principais tratamentos descritos para a perda de peso foram a realização de mudanças na alimentação por conta própria $(65,4 \%)$, dieta e prática de atividade física $(42,3 \%)$ e uso de medicação (26,9\%). O acompanhamento nutricional foi pouco citado $(34,6 \%)$ como método para perda de peso. Observa-se que houve superposição das práticas descritas acima pelas pacientes.

\section{Discussão}

No presente estudo, constatou-se que as classificações do IMC dos pesos aferido, habitual e desejado são diferentes entre si. Interessantemente, as pacientes chegam ao serviço com obesidade grau II, que é superior à classificação do IMC habitual relatado (obesidade grau I), e desejam atingir IMC classificado como sobrepeso, ou seja, inferior ao IMC habitual.

Tendo em vista que majoritariamente as pacientes apresentaram peso aferido superior ao habitual, busca-se compreender a relação desse ganho de peso com os marcos temporais e a trajetória da flutuação de peso. É importante destacar que os marcos temporais são eventos que promovem mudanças no contexto social e alimentar do paciente, como, por exemplo, a alteração na rotina de trabalho e acometimento de doença e a gravidez. ${ }^{25-27}$

Um marco temporal recorrente nos relatos foi o casamento, que também foi referido como um preditor de ganho de peso por Teachman ${ }^{28}$ e Klos \& Sobal. ${ }^{29}$ Os problemas familiares também podem influenciar no ganho de peso, como divórcio e morte do cônjuge. ${ }^{30}$

Além dos marcos temporais de peso, a presença de excesso de peso desde a infância foi salientada e concorda com dados publicados por Matos et al., ${ }^{31}$ os quais associam o sobrepeso e obesidade em adultos com excesso de peso na primeira infância. 
O entendimento da história de ganho de peso é indispensável ao tratamento e pode promover uma abordagem mais adequada às demandas e vivências do paciente. ${ }^{32}$ Esses resultados sinalizam o papel da alimentação como um marcador do modo de vida e alerta serviços de saúde e outros para a necessidade de abordar esses períodos de maneira a prevenir o aumento do peso corporal.

Analisando a história de peso das pacientes deste estudo, observa-se que a perda seguida de ganho de peso $(77,0 \%)$ é uma condição frequente no grupo estudado. Entre os americanos, quase metade da população tenta perder peso, entre os que conseguem, a maioria ganha peso novamente. ${ }^{33}$ Em uma coorte de mais de 3.500 mulheres, $10 \%$ apresentavam peso flutuante severo (três perdas de peso superior a $5 \mathrm{~kg}$ com posterior ganho). ${ }^{21}$ Uma pesquisa brasileira, com mulheres atendidas em um ambulatório de obesidade, encontrou peso flutuante em 59,1\% das mulheres. Segundo os autores, essa oscilação de peso pode dificultar a adesão ao tratamento e elevar o índice de abandono. ${ }^{20}$ Oliveira et al., por outro lado, observaram o peso flutuante em apenas $13 \%$ dos pacientes estudados. Esta porcentagem inferior de pacientes com peso flutuante pode estar associada ao relato de uso de fármacos $(84,2 \%)$ dos pacientes e/ou por se tratar de um ambulatório pré-cirurgia bariátrica, fator que pode motivar os pacientes à perda de peso. ${ }^{22}$

A maioria das pacientes deste estudo $(65,8 \%)$ desejavam perder entre $25 \%$ e $50 \%$ do peso aferido, o que coincide com os dados publicado por Grave, nos quais 1.011 pacientes esperavam uma perda de $9 \mathrm{~kg} / \mathrm{m}^{2}$ de IMC em um ano de tratamento e desejavam um peso $32 \%$ menor que o inicial. Nesse mesmo estudo, quando questionados sobre um IMC máximo considerado aceitável, foi relatado uma média de peso $23 \%$ menor que o atual. ${ }^{2}$ Todavia, o estudo de Befort et al. mostrou que as mulheres desejavam uma perda média de18,8\% do peso inicial, ou seja, média inferior aos achados deste estudo. ${ }^{15}$

Considerando que a grande maioria das pacientes (99,3\%) apresentaram desejo de perda expressiva de peso aliada a tentativas prévias ineficazes e a movimentos de reganho de peso, salienta-se a possibilidade da insatisfação corporal. Estudos mostram que mais de $60 \%$ das mulheres obesas relatam insatisfação com o peso corporal. ${ }^{34,35}$

Algumas vezes, a insatisfação corporal pode motivar a busca por tratamento de perda de peso para, além de aplacar questões de saúde, adaptar-se às imposições sociais e padrões estéticos vigentes naquele grupo social. ${ }^{2}$

Apesar de os padrões de beleza e ideais estéticos estarem apresentados no discurso das mulheres, muitas vezes eles são moldados por diversos fatores externos ao sujeito. Um fator que pode moldar essa idealização do corpo é a própria dinâmica dos ideais estéticos do corpo em cada contexto social e histórico. ${ }^{11,19}$ Nesse sentido, um estudo inglês, ao analisar uma população em 1999 e em 2007, associou o aumento da prevalência da obesidade com a redução da percepção de excesso de peso pelos sujeitos estudados. ${ }^{11}$ 
A mídia também tem um papel central que influencia a construção de padrões corporais na sociedade contemporânea. ${ }^{36} \mathrm{O}$ sujeito é exposto diariamente à televisão, filmes, revistas e internet que mostram imagens de modelos e corpos em um ideal de beleza longe do apresentado pela maioria da população. ${ }^{3}$ Esses ideais culturais podem moldar a percepção pública do ideal do corpo feminino. A mídia tem um papel fundamental no aumento da insatisfação com o próprio corpo. ${ }^{36}$ Por exemplo, a taxa de exposição a novelas e filmes é associada com maior insatisfação corporal em adolescentes. ${ }^{37}$

A discrepância entre o peso habitual e o peso desejado, assim como os diferentes achados relacionados aos marcos temporais de ganho de peso e às trajetórias da história corporal das pacientes deste estudo, integra-se às informações sobre a influência do contexto social e da mídia descrita na literatura. Nota-se a diversidade de fatores que influenciam na construção social do corpo para a paciente com excesso de peso. Essas diferentes construções podem influenciar a aderência às recomendações para perda de peso, pois a motivação pessoal do paciente está estreitamente ligada com a percepção do próprio peso corporal. ${ }^{14,18}$

\section{Conclusão}

Este estudo mostrou que há uma discrepância entre o peso aferido, o peso habitual e o peso desejado pelas mulheres que buscam tratamento nutricional para perda de peso. Além disso, foi observado diversos fatores que influenciam a construção da história de peso da paciente: diferentes marcos temporais de ganho de peso, tentativas de perda de peso prévias de tratamento e flutuações de peso no decorrer da vida. Desse modo, mostra-se pertinente investigar, entender a história de peso de cada paciente no contexto clínico do tratamento nutricional, pois essas informações podem servir de parâmetro para estabelecer metas mais eficazes e melhorar a adesão ao tratamento para perda de peso.

\section{Agradecimentos}

Agradecemos a toda a equipe do ambulatório, Camila Cremonezi Japur, Fernanda Rodrigues de Oliveira Penaforte, Flávia Gonçalves Micali. Agradecemos ao CNPq, pelo,fomento à pesquisa de bolsa de produtividade de RWDG. 


\section{Referências}

1. World Health Organization. Obesity: preventing and managingthe global epidemia. Report of a World Health Organization Consultation. Geneva: WHO; 2000. 256 p. WHO Obesity Techinical Report Series.

2. Dalle Grave R, Calugi S, Magri F, Cuzzolaro M, Dall'aglio E, Lucchin L, et al. Weight loss expectations in obese patients seeking treatment at medical centers. Obes Res. 2004; 12(12):2005-12.

3. Campos MTA, Cecília MS, Penaforte FRO. Corpo-vitrine, ser mulher e saúde: produção de sentidos nas capas da Revista Boa Forma. Demetra 2016; 11:611-28.

4. Araújo CL, Dumith SC, Menezes AMB, Hallal PC. Measured weight, self-perceived weight, and associated factors in adolescents. Rev Panam Salud Publica 2010; 27(5):360-7.

5. National Heart, Lung, and Blood Institute. Obesity Education Initiative Expert Panel on the Identification E, and Treatmentof Obesity in Adults (US). Clinical guidelines on the identification, evaluation, and treatment of overweight and obesity in adults. Bethesda, MD: NHLBI; 1998.

6. Blackburn G. Effect of degree of weight loss on health benefits. Obes Res. 1995; 3(Suppl 2):211s-16s.

7. Goldstein DJ. Beneficial health effects of modest weight loss. Int J Obes Relat Metab Disord. 1992; 16(6):397-415.

8. Kanders B, Blackburn G. Reducing primara risk factors by therapeutic weight loss. In: Wadden TA, van Itallie TB, editor. Treatment of seriously obese patients. New York: The Guildford Press, 1992. p. 213-30.

9. Johnson F, Cooke L, Croker H, Wardle J. Changing perceptions of weight in Great Britain: comparison of two population surveys. BMJ 2008; 337(7664):a494.

10. Vigarello G, Penchel M. As metamorfoses do gordo: história da obesidade no Ocidente; da Idade Média ao século XX. Petropolis: Vozes; 2012.

11. Christakis NA, Fowler JH. The spread of obesity in a large social network over 32 years. N Engl J Med. 2007; 357(4):370-9.

12. Maynard LM, Serdula MK, Galuska DA, Gillespie C, Mokdad AH. Secular trends in desired weight of adults. International Journal of Obesity 2006; 30(9):1375-81.

13. Foster GD, Wadden TA, Vogt RA, Brewer G. What is a reasonable weight loss? Patients' expectations and evaluations of obesity treatment outcomes. J Consult Clin Psychol. 1997; 65(1):79-85.

14. Duncan DT, Wolin KY, Scharoun-Lee M, Ding EL, Warner ET, Bennett GG. Does perception equal reality? Weight misperception in relation to weight-related attitudes and behaviors among overweight and obese US adults. Int J Behav Nutr Phys Act. 2011; 8:20.

15. Befort CA, Allen Greiner K, Hall S, Pulvers KM, Nollen NL, Charbonneau A, et al. Weight-related perceptions among patients and physicians: how well do physicians judge patients' motivation to lose weight? J Gen Intern Med. 2006; 21(10):1086-90.

16. Elfhag K, Rossner S. Who succeeds in maintaining weight loss? A conceptual review of factors associated with weight loss maintenance and weight regain. Obes Rev. 2005; 6(1):67-85. 
17. Cooper Z, Fairburn CG. A new cognitive behavioural approach to the treatment of obesity. Behav Res Ther. 2001; 39(5):499-511.

18. Fan M, Jin Y. The effects of weight perception on adolescents' weight-loss intentions and behaviors: evidence from the youth risk behavior surveillance survey. Int J Environ Res Public Health 2015; 12(11):14640-68.

19. Almeida GAN, Santos JE, Pasian SR, Loureiro SR. Percepção de tamanho e forma corporal de mulheres: estudo exploratório. Psicologia em Estudo 2005; 10(1):27-35.

20. Andrade BMC, Mendes CMC, Araújo LMB. Weight cycling during treatment of obese women. Peso flutuante no tratamento de mulheres obesas. Arq Bras Endocrinol Metab. 2004; 48(2):276-81.

21. Lahti-Koski M, Mannisto S, Pietinen P, Vartiainen E. Prevalence of weight cycling and its relation to health indicators in Finland. Obes Res. 2005; 13(2):333-41.

22. Oliveira SPSG, Pecis M, Aveline MK, Schmidt AC, Zaslavsky LMA, Souto KEP, et al. Flutuação de peso nos pacientes em tratamento de obesidade atendidos no ambulatório de endocrinologia do Hospital Nossa Senhora da Conceição entre 2002 e 2005. Revista da AMRIGS 2007; 3:190-7.

23. Schulz M, Liese AD, Boeing H, Cunningham JE, Moore CG, Kroke A. Associations of short-term weight changes and weight cycling with incidence of essential hypertension in the EPIC-Potsdam Study. J Hum Hypertens. 2005; 19(1):61-7.

24. Braun V, Clarke V. Using thematic analysis in psychology. Qualitative Research in Psychology 2006; 3:77-101.

25. Ponteiro FM, Bachion MM. Crenças de pacientes diabéticos acerca da terapia nutricional e sua influência na adesão ao tratamento. Ciênc Saúde Coletiva 2010; 15(1):151-60.

26. Toral N, Slater B. Abordagem do modelo transteórico no comportamento alimentar. Ciênc Saúde Coletiva 2007; 12:1641-50.

27. Viana V. Psicologia, saúde e nutrição: contributo para o estudo do comportamento alimentar. Análise Psicológica 2002; 4:611-24.

28. Teachman J. Body weight, marital status, and changes in marital status. J Fam Issues 2016; 37(1):74-96.

29. Klos LA, Sobal J. Marital status and body weight, weight perception, and weight management among U.S. adults. Eat Behav. 2013; 14(4):500-7.

30. Umberson D, Crosnoe R, Reczek C. Social relationship and health behavior across life course. Annu Rev Social 2010; 36:139-57.

31. Matos SMA, Jesus SR, Saliva SRDM, Prado MS, D’Innocenzo S, Assis AMO, et al. Velocidade de ganho de peso nos primeiros anos de vida e excesso de peso entre 5-11 anos de idade, Salvador, Bahia, Brasil. Cad Saúde Pública 2011; 27:714-22.

32. Fonseca MJM, França RF, Faerstein E, Werneck GL, Chor D. Escolaridade e padrões de ganho de peso na vida adulta no Brasil: Estudo Pró-Saúde. Rev Panam Salud Pública 2012; 32(5):376-80.

33. Kroke A, Liese AD, Schulz M, Bergmann MM, Klipstein-Grobusch K, Hoffmann K, et al. Recent weight changes and weight cycling as predictors of subsequent two year weight change in a middleaged cohort. Int J Obes 2002; 26(3):403-9. 
34. Sarwer DB, Wadden TA, Foster GD. Assessment of body image dissatisfaction in obese women: specificity, severity, and clinical significance. J Consult Clin Psychol. 1998; 66(4):651-54.

35. Cheung YTD, Lee, Ho SY, Li ETS, Lam TH, Fan SYS, et al. Who wants a slimmer body? The relationship between body weight status, education level and body shape dissatisfaction among young adults in Hong Kong. BMC Public Health 2011; 11:835.

36. Derenne JL, Beresin EV. Body image, media, and eating disorders. Acad Psychiatry 2006; 30(3):257-61.

37. Tiggemann M, Australia TFUoS, The Flinders University of South Australia GPOB, Adelaide 5001, South Australia, Pickering AS, Australia CPatUoS. Role of television in adolescent women's body dissatisfaction and drive for thinness. Int J Eating Disorders 2016; 20(2):199-203.

Recebido: $15 / 4 / 2016$

Revisado: $18 / 9 / 2016$

Aceito: 03/11/2016 